\title{
Metabolic Balance Cot for the Newborn Infant
}

Dr. Colin H. M. WALKER, consultant paediatrician, Dundee Royal Infirmary and the Department of Child Health, University of Dundee, writes: Techniques for the performance of metabolic balance studies in infants can be divided into two main categories-those in which diaper collection is used (Slater, 1961 ; Widdowson, 1965) and those in which a special cot with a catchmen area is employed (Forfar and Prain, 1950 ; Barness et al., 1957). A disadvantage of the first method is the risk of loss from a loose diaper or leakage of soft stool past a displaced plastic liner. Balances which require the application of cups (Thomson, 1944) or adhesive bags (Baldwin et al., 1962) have the disadvantages of skin trauma, and constant observation by nursing staff is required to ensure adequate application of the collector. In fact it has been said that "success ultimately depends upon the persistent and personal supervision of the collections by members of the team, who must be prepared to devote a considerable part of their time solely to this aspect of the study" (Stevens et al., 1962).

As medical and nursing staff become more and more difficult to obtain there is a reluctance to ask staff to take part in those metabolic balance studies which might be regarded as yielding useful information yet nonessential to immediate patient-care. For the purpose of performing total metabolic balances of fat and calcium in the newborn an alternative method of collection has therefore been designed.

\section{Metabolic Cot}

The cot consists of two main parts which can be used in conjunction with a standard folding crib currently in use in many nurseries (Fig. 1). The outer part consists of a V-shaped catchment which declines to the feet and drains into a funnel at the foot end

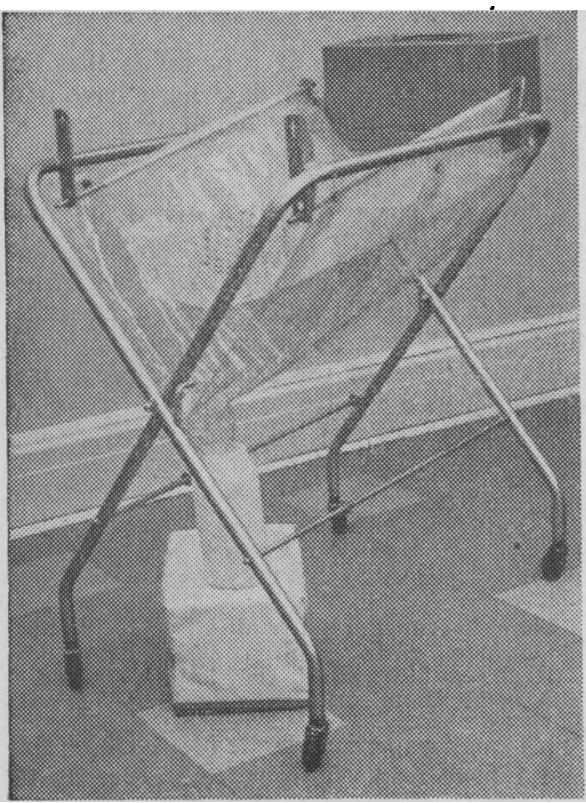

FIG. 1.-Metabolic cot assembled without covers.

of the cot. It is made of $0.01-\mathrm{in}$. $(0.25 \mathrm{~mm}$.) transparent polyvinyl chloride plastic and is electronically welded at the edges in such a way that cleaning is not difficult. The inner layer is suspended on a second pair of rods and is a sling of $0.02-\mathrm{in}$. $(0.5-\mathrm{mm}$.) opaque polyvinyl chloride in which $\frac{3}{8}$-in. $(1-\mathrm{cm}$.) holes have been punched $\frac{5}{8}-$ in. $(1.6-\mathrm{cm}$.) apart (Fig. 2). The holes are punched in such a way that there is slight embossing with the sharp edge pointing downwards, and though the skin is marked by the holes no injury or

the infants behave normally while nursed on the sling and are fully observable. The assembly is inexpensive, is easily cleaned, and sustains contact with detergent and chemical antiseptics and cleaners. A standard ward cot can be employed and the catchment and sling can be stored in a small space when not in use.

The disadvantages include the need for a temporary napkin collection during breastfeeding, though the advantages remain between whiles. If separate stool and urine

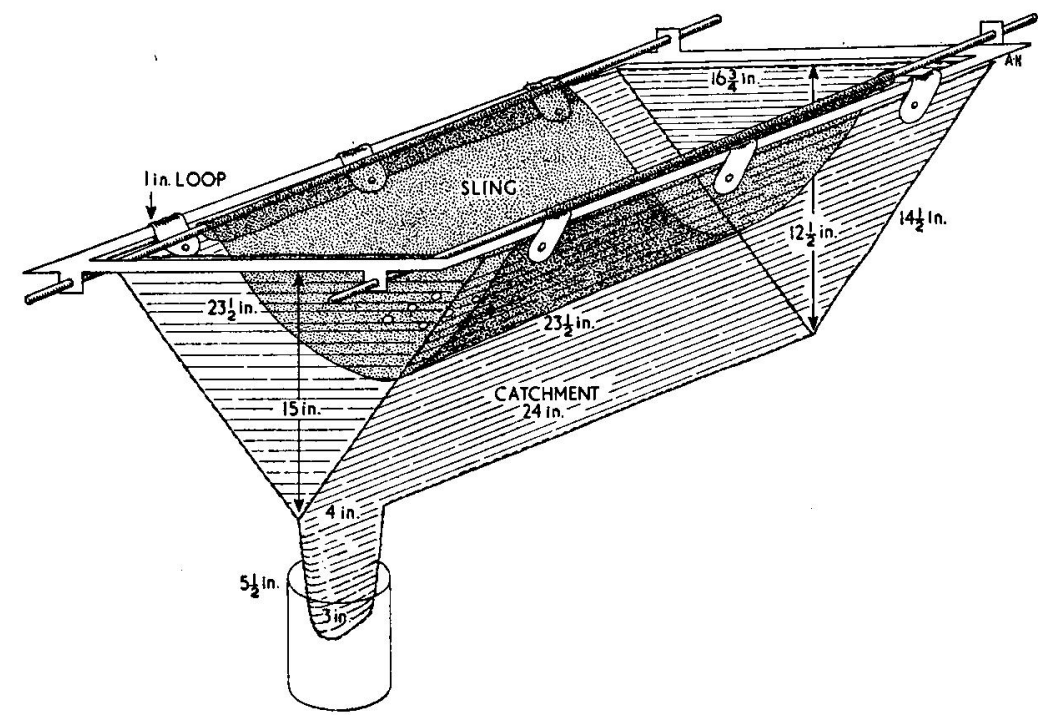

FIG. 2.-Diagram of metabolic cot with dimensions.

irritation occurs over the balance period. This material was selected after other pure plastics and plasticized fibres had been found unsatisfactory because of skin trauma, lack of strength, fraying, or absorption of fluid by fibres.

The cot blanket is encased in nonabsorbent plastic and the infant is nursed naked on the sling. The body temperature falls no more than $1^{\circ} \mathrm{F}$. $\left(0.55^{\circ} \mathrm{C}\right.$.) in nursery kept at an average temperature of $78^{\circ}$ F. $\left(25.6^{\circ}\right.$ C. $)$. All excreta either run through the sling on to the catchment or can be washed through. Extra firm stools may be picked off the sling, but as the stool of a newborn infant is usually soft this is seldom necessary. Collection is made into polyethylene bottles continuously mounted below the funnel, and both cot and baby can be swabbed down with deionized water and brush nylon wipes as often as required. The infant is fed in the cot by nurse or mother. During feeding a non-absorbent bib of nylon seersucker is used to catch any regurgitation or vomitus, just as in other study methods, and any wipes used can also be kept in a separate polyethylene bottle for analysis and correction of " intake."

The advantages of this cot are that it allows total collection with minimal handling of the baby and minimal nursing care. The method is fully acceptable by mothers, and collection is required an adhesive bag technique can be added, and should leakage occur it would probably be more readily noticed in the "open" cot than with a "closed" diaper technique. Occasionally a rather large quantity of deionized water is required for thorough cleansing at the end of the balance study. This makes the analysis more tedious but none the less efficient.

Thanks are expressed to Mr. L. A McKenzie and Mr. W. Kay, Regional Physics Laboratory, Eastern Regional Hospital Board, Scotland, and Mr. D. M. High, of D. M. High, Waterproof Clothing Manufacturers, Dundee, for their assistance in the construction of this metabolic cot.

This project is supported by a grant from John Wyeth \& Brother Ltd.

\section{REFERENCES}

Baldwin, E. M., Clayton, B. E., Jenkins, P. Mitchell, J.", and Renwick, A. G. C. (1962) Barness. L. A., Baker, D.' Guilbert, P. Torrea,
F. E., and György, P.' (1957). 尹. Pedial., E1;

Forfar, J. O., and Prain, J. H. (1950). Arch. Dis. Childh., 25, 260.

Slater, J. E. (1961). Brit. 7. Nutr., 15, 83. Stevens, L. H., Hughes, E. A., and Wilkinson Thomson, J. (1944). Ibid, 19, 169.

Widdowson, B. M. (1965). Lancet, 2, 1099. 